\title{
Feasibility of 5-days-on/2-days-off UFT/leucovorin in post-operative long-term adjuvant chemotherapy for colorectal cancer
}

\author{
YUKIHIKO TOKUNAGA and HIROKAZU SASAKI \\ Department of Surgery, Osaka North Japan Post Hospital, Osaka, Japan \\ Received November 24, 2011; Accepted January 30, 2012
}

DOI: $10.3892 / 01.2012 .590$

\begin{abstract}
Previous clinical studies have shown that the oral uracil/tegafur (UFT)/leucovorin (LV) regimen, in which the drugs are taken for 28 consecutive days every 35 days, is equivalent to an infusional 5-fluorouracil (5-FU)/LV regimen for the treatment of colorectal cancer. A 5-days-on/2-daysoff schedule for UFT/LV has been proposed as the same schedule for UFT has been reported to be safe and have good compliance. However, few studies have been performed with regards to the feasibility of the UFT/LV regimen. The results of the 5-days-on/2-days-off schedule were compared with those of the consecutive schedule in adjuvant chemotherapy. Twenty-eight patients were treated with the 5-days-on/ 2-days-off schedule of UFT (300 mg/m²/day)/LV (75 mg/ body/day), and another 12 patients were treated with the consecutive schedule. In the 5-days-on/2-days-off schedule, 24 of 28 patients $(86 \%)$ received all the scheduled doses. In the consecutive schedule, 10 of 12 patients $(83 \%)$ received all the scheduled doses. The mean relative dose intensities for the 5-days-on/2-days-off and consecutive schedules were 0.92 and 0.87 , respectively. The toxicities were milder in the 5-days-on/2-days-off schedule compared with the consecutive schedule. The disease-free survival in patients with the 5-days-on/2-days-off schedule tended $(\mathrm{P}=0.13)$ to be longer compared with the consecutive schedule. The results of the present study indicate that the 5-days-on/2-days-off schedule of UFT/LV may be feasible and cause no severe toxicities in long-term adjuvant chemotherapy.
\end{abstract}

Correspondence to: Dr Yukihiko Tokunaga, Department of Surgery, Osaka North Japan Post Hospital, 1-1-6 Nakazaki, Kita-ku, Osaka 530-8798, Japan

E-mail: tokunagay@usay6.com

Abbreviations: UFT, uracil/tegafur; LV,leucovorin; CRC, colorectal cancer

Key words: colorectal cancer, chemotherapy, UFT/leucovorin

\section{Introduction}

Previous clinical studies have shown that the conventional oral uracil/tegafur (UFT)/leucovorin (LV) regimen, in which the drugs are taken for 28 consecutive days every 35 days, is equivalent to an infusional 5-fluorouracil (5-FU)/LV regimen for the treatment of colorectal cancer (CRC) $(1,2)$. The conventional UFT/LV regimen has been accepted as one of the adjuvant chemotherapies following surgery. A 5-day-on/2-day-off schedule for UFT/LV, which is followed on 5 weekdays followed by 2 weekend days off, has been proposed as the same schedule for UFT has been reported to be safe and have good compliance (3-5). However, few studies have been performed with regards to the feasibility of the 5-days-on/2-days-off schedule of UFT/LV in terms of safety and compliance. The aim of the present study was to assess the feasibility of the schedule in long-term adjuvant chemotherapy for CRC patients as compared with the consecutive schedule of the UFT/LV regimen.

\section{Patients and methods}

Patients. Between January 2006 and June 2009, 40 patients were treated with oral UFT/LV as adjuvant chemotherapy following surgery for CRC. Patients were prospectively allocated to one of the two UFT/LV schedules at a 2:1 ratio. Of the 40 patients, 28 were treated with UFT $\left(300 \mathrm{mg} / \mathrm{m}^{2} /\right.$ day $) / \mathrm{LV}$ ( $75 \mathrm{mg} /$ body/day) which was taken on 5 weekdays followed by 2 weekend days off. One course lasted 5 weeks and 15 courses (75 weeks) were administered. The remaining 12 patients were treated with UFT (300 mg/m²/day)/LV ( $75 \mathrm{mg} /$ body/day) which was taken for 28 consecutive days every 35 days according to the conventional schedule. One course lasted 5 weeks ( 35 days) and 5 courses (25 weeks) were administered. We compared the results of the two schedules in terms of safety and compliance.

The study was approved by the ethics committee of our hospital as well as the Japanese Foundation for Multidisciplinary Treatment of Cancer. Consent was obtained from each patient prior to the start of the study.

Toxicities. The toxicities were graded according to the common terminology criteria for adverse events v 3.0 (CTCAE) (6). 
Table I. Patient characteristics.

\begin{tabular}{lc}
\hline Characteristic & Number of patients \\
\hline Number of cases & 40 \\
Age, years (mean \pm SD) & $67 \pm 7.6$ \\
Gender & \\
Male & 24 \\
Female & 16 \\
Location of cancer & \\
Colon & 27 \\
Rectum & 13 \\
Stage & \\
II & 20 \\
III & 20 \\
Performance status & \\
0 & 37 \\
1 & 3 \\
Pathology & \\
Well-diff adeno & 14 \\
Moderately diff adeno & 26 \\
\hline
\end{tabular}

diff adeno, differentiated adenocarcinoma.

Table II. Treatment compliance.

\begin{tabular}{lcc}
\hline & $\begin{array}{c}\text { 5-days-on/2-days-off } \\
\text { schedule } \\
\mathrm{n}=28\end{array}$ & $\begin{array}{c}\text { Consecutive } \\
\text { schedule } \\
\mathrm{n}=12\end{array}$ \\
\hline Treatment course & & \\
Mean \pm SD & $13.8 \pm 3.2$ & $4.7 \pm 0.2$ \\
Median (range) & $15(2-15)$ & $5(3-5)$ \\
Completion & $24(86 \%)$ & $10(83 \%)$ \\
Dose reduction & $3(11 \%)$ & $2(17 \%)$ \\
Discontinuation & $4(14 \%)$ & $2(17 \%)$ \\
Relative dose density & & \\
Mean \pm SD & $0.92 \pm 0.2$ & $0.87 \pm 0.2$ \\
Median (range) & $1(0.15-1)$ & $1(0.4-1)$ \\
\hline
\end{tabular}

5-days-on/2-days-off schedule, UFT (300 mg/m²/day)/LV (75 mg/ body/day) taken on 5 weekdays followed by 2 weekend days off; consecutive schedule, UFT (300 mg/m²/day)/LV (75 mg/body/day) taken for 28 consecutive days every 35 days.

In the case of hematologic or non-hematologic toxicities of grade 2 or above, the UFT/LV regimen was occasionally stopped following consultation with the patient. If hematologic or non-hematologic toxicities of grade 2 developed, the UFT dose was reduced for the subsequent course. The patients were free to reject chemotherapy for any reason.

Statistical analysis. Statistical analyses were performed using non-parametric methods including the Chi-square test and

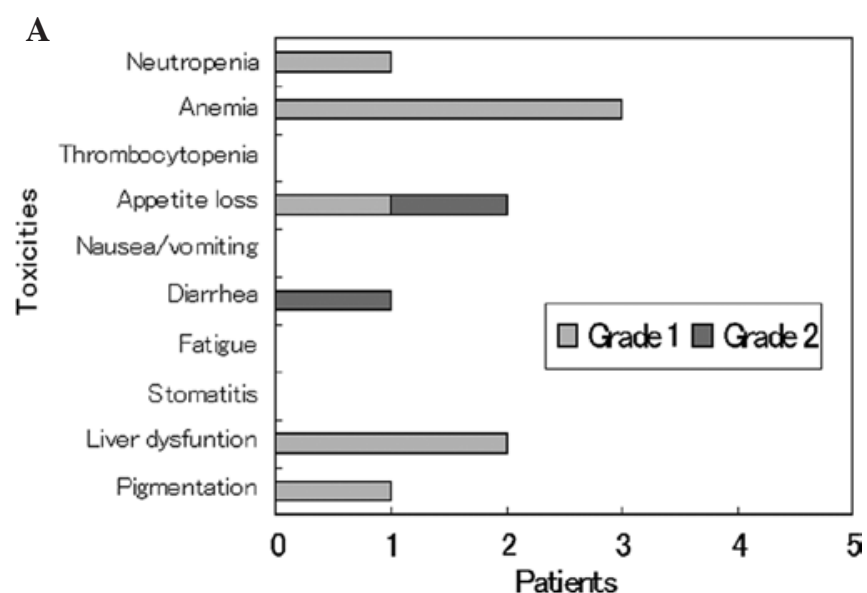

B

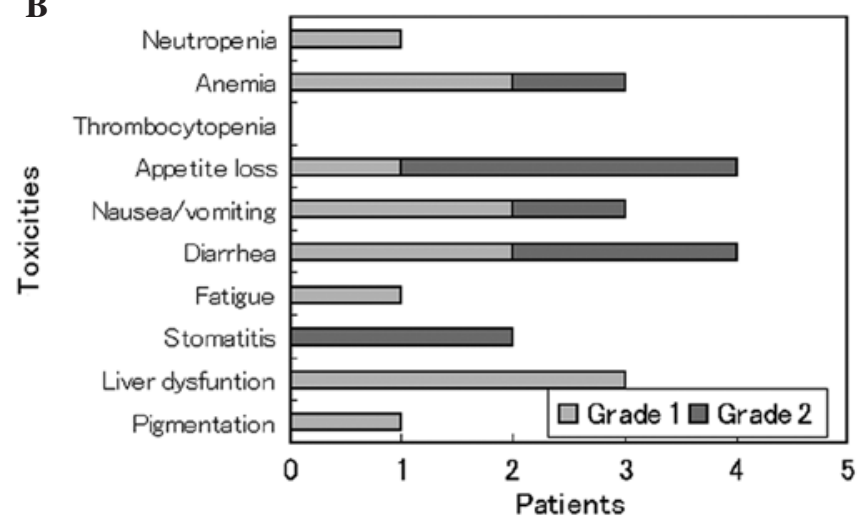

Figure 1. (A) Toxicities, overall number of patients who experienced toxicities in the 5-days-on/2-days-off schedule of UFT/LV $(n=28)$. (B) Toxicities, overall number of patients who experienced toxicities in the consecutive schedule of UFT/LV $(n=12)$.

Fisher's exact probability test. Survival curves were calculated using the Kaplan-Meier method and differences were evaluated with the log-rank test. $\mathrm{P}<0.05$ was considered to indicate a statistically significant result.

\section{Results}

Patients and treatment courses. Patient characteristics included a mean age of 67 years (range 55-79) (Table I). Patients had a performance status of 0 or 1 . Rectal cancer patients accounted for $32.5 \%$ of the total number of patients. In the 5-days-on/2-days-off treatment schedule, 24 of 28 patients $(86 \%)$ received all the scheduled doses of the regimen (Table II). The mean relative dose intensity was 0.92 and the mean number of treatment courses administered was 13.8. In the consecutive schedule, 10 of 12 patients $(83 \%)$ received all the scheduled doses of the regimen. The mean relative dose intesity was 0.87 and the mean number of treatment courses administered was 4.7 .

Toxicities and compliance. The toxicities were mild and no patient experienced a toxicity of grade 3 or 4 in either schedule. A total of 10 patients experienced toxicities in the 5-days-on/2-days-off schedule (Fig. 1A) and a total of 22 patients experienced toxicities in the consecutive schedule (Fig. 1B). There were fewer patients with grade 2 toxicities 


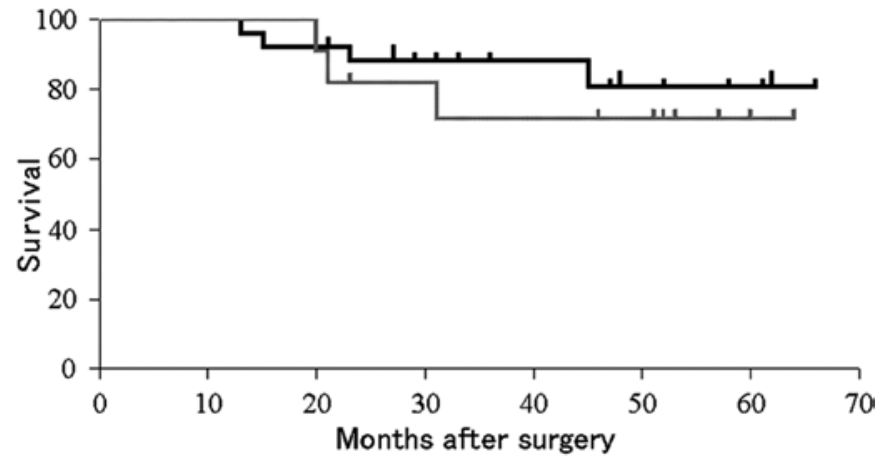

Figure 2. Disease-free survival in patients with the 5-days-on/2-days-off schedule (black line) tended $(\mathrm{P}=0.13)$ to be longer than those with the consecutive schedule (gray line).

$(\mathrm{P}<0.01)$ in the 5-days-on/2-days-off schedule compared to the consecutive schedule. The toxicities were milder in the 5-days-on/2-days-off schedule compared to the consecutive schedule. There were 6 cases of discontinuation of the regimens, 4 in the 5-days-on/2-days-off schedule and 2 in the consecutive schedule. The reasons for discontinuation were recurrence ( 2 cases), toxicities ( 2 cases) and patient rejection ( 2 cases). The disease-free survival in patients with the 5-days-on/2-days-off schedule tended $(\mathrm{P}=0.13)$ to be longer than those with the consecutive schedule (Fig. 2).

\section{Discussion}

Previous clinical studies have shown that the consecutive UFT/LV regimen, in which the drugs are taken for 28 consecutive days every 35 days, is equivalent to an infusional 5-FU/LV regimen for CRC treatment $(1,2)$. The consecutive UFT/LV regimen has been widely accepted as adjuvant chemotherapy in Japan. Concerning the consecutive regimen, Douillard et al reported that non-hematological toxicities were diarrhea $(21 \%)$ and nausea/vomiting (13\%) (1). Carmichael et al reported that diarrhea and nausea/vomiting developed in 18 and $9 \%$ of patients, respectively (2).

In the National Surgical Adjuvant Breast and Bowel Project (NSABP) C-06 clinical trial, the consecutive UFT/LV regimen was used as an adjuvant chemotherapy (7). Grade 3 or above diarrhea developed in $29 \%$ of patients and vomiting in $5 \%$. Meguro et al reported that the consecutive UFT/LV regimen could be completed in $82.5 \%$ of patients without any toxicity of grade 3 or above (8). The authors reported, however, that the most frequent non-hematologic toxicities were appetite loss (50\%) and fatigue $(60 \%)$ and that diarrhea and nausea/vomiting developed in 30 and $40 \%$ of patients, respectively. The results of the present study concerning the 5-days-on/2-days-off schedule of UFT/LV revealed only grade 2 appetite loss (4\%) and diarrhea (4\%) without any toxicity of grade 3 or above.

In the previous chemotherapy, with UFT as a single agent, the 5-days-on/2-days-off schedule has been reported to be safer and more feasible than the consecutive schedule (5). Tegafur is metabolized to 5-FU by the hepatic P-450 drug-metabolizing enzyme. 5-FU is known to be involved in two mechanisms of anticancer action, concentration-dependent RNA dysfunction and time-dependent DNA synthesis inhibition (10). As the 5-days-on/2-days-off schedule of UFT/LV was able to provide a relatively high dose of UFT for a longer duration than the consecutive schedule $(4,5)$, the antitumor effects might be more marked in the 5-days-on/2-days-off schedule compared to the consecutive schedule.

Few studies that have evaluated the optimal duration of adjuvant chemotherapy for CRC are currently available $(11,12)$. With infusional 5-FU/LV regimens, a duration of 6 months has been recommended on the basis of toxicities, cost and convenience of the patients, since previous studies found no significant difference in disease-free survival and/or overall survival between the patients treated with adjuvant chemotherapy for 6 months and those treated for 12 months $(11,12)$. However, with oral tegafur regimens, several clinical studies have indicated the efficacy of adjuvant chemotherapy for 1-2 years $(13,14)$. As recurrence occurs a year following curative resection in $\sim 30 \%$ of high-risk patients (15), it would be useful to evaluate the feasibility of oral UFT/LV in post-operative long-term adjuvant chemotherapy.

The results of the present study indicate that the toxicities were milder in the 5-days-on/2-days-off schedule of UFT/LV as compared to the consecutive schedule. The 5-days-on/2days-off schedule of UFT/LV may thus be feasible and cause no severe toxicities in post-operative long-term adjuvant chemotherapy for CRC.

\section{References}

1. Douillard JY, Hoff PM, Skillings JR, Eisenberg P, Davidson N, Harper P, Vincent MD, Lembersky BC, Thompson S, Maniero A and Benner SE: Multicenter phase III study of uracil/tegafur and oral leucovorin versus fluorouracil and leucovorin in patients with previously untreated metastatic colon cancer. J Clin Oncol 20: 3605-3616, 2002.

2. Carmichael J, Popiela T, Radstone D, Falk S, Borner M, Oza A, Skovsgaard T, Munier S and Martin C: Randomized comparative study of tegafur/uracil and oral leucovorin versus parenteral fluorouracil and leucovorin in patients with previously untreated metastatic colorectal cancer. J Clin Oncol 20: 3617-3627, 2002

3. Sadahiro S, Mukai M, Tokunaga N, Tajima T, Makuuchi H, Yoshida M, Okabe H, Uchida J, Takeda S and Unemi N: Preliminary study on the optimal dosage schedule for oral tegafur/uracil(UFT) chemotherapy. Int J Clin Oncol 3: 7-12, 1998.

4. Sadahiro S, Suzuki T, Kameya T, Iwase H, Tajima T and Makuuchi H: A pharmacological study of the weekday-on/ weekend-off oral UFT schedule in colorectal cancer patients. Cancer Chemother Pharamacol 47: 457-460, 2001.

5. Sadahiro S, Ohki S, Yamaguchi S, Takahashi T, Otani Y, Tsukikawa S, Yamamura T, Takemiya S, Nagaski $H$, Nishiyama K, et al: Feasibility of a novel weekday-on/ weekend-off oral UFT schedule as postoperative adjuvant chemotherapy for colorectal cancer. Cancer Chemother Pharmacol 46: 180-184, 2000.

6. Japanese translation of common terminology criteria for adverse events (CTCAE), and instructions and guidelines. Int J Clin Oncol 9 (Suppl 3): 1-64, 2004 (In Japanese).

7. Lembersky BC, Wieand HS, Petrelli NJ, O'Connell MJ, Colangelo LH, Smith RE, Seary TE, Giguere JK, Marshall ME, Jacobs AD, et al: Oral uracil and tegafur plus leucovorin compared with intravenous fluorouracil and leucovorin in stage II and III carcinoma of the colon: Results of national surgical adjuvant breast and bowel project protocol C-06. J Clin Oncol 24: 2059-2064, 2006.

8. Meguro M, Furuhata T, Okita K, Nishidate T, Ishiyama G, Iwayama Y, Kimura Y, Mizuguchi T and Hirata K: Clinical compliance with oral uracil/tegafur (UFT) plus leucovorin (LV) regimen as adjuvant chemotherapy in Japanese colorectal cancer patients. Int J Clin Oncol 14: 402-407, 2009. 
9. Inaba M and Mitsuhashi Y: Flow cytometric analysis of cell-killing action of 5-fluorouracil in human colorectal cancer cells. Oncol Res 6: 303-309, 1994.

10. Sadahiro S, Suzuki T, Ishikawa K, Nakamura T, Saguchi T, Kamijyo A, Yasuda S, Makuuchi H and Murayama C: Preliminary study on the optimal dosing schedule for oral UFT/leucovorin chemotherapy. Anticancer Res 24: 625-630, 2004.

11. O'Connell MJ, Laurie JA, Kahn M, Fitzgibbons RJ Jr, Erlichman C, Shepherd L, Moertel CG, Kocha WI, Pazdur R, Wieand HS, et al: Prospective randomized trial of postoperative adjuvant chemotherapy in patients with high-risk colon cancer. J Clin Oncol 16: 295-300, 1998.

12. Haller DG, Catalano PJ, MacDonald JS, O'Rourke MA, Frontiera MS, Jackson DV and Mayer RJ: Phase III study of fluorouracil, leucovorin, and levamisole in high-risk stage II and III colon cancer: final report of Intergroup 0089. J Clin Oncol 23 : 8671-8678, 2005.
13. Kato T, Ohashi Y, Nakazato H, Koike A, Saji S, Suzuki H, Takagi H, Nimura Y, Hasumi A, Baba S, et al: Efficacy of oral UFT as adjuvant chemotherapy to curative resection of colorectal cancer: Multicenter prospective randomized trial. Langenbecks Arch Surg 386: 575-581, 2002.

14. Sakamoto J, Ohashi Y, Hamada C, Buyse M, Burzykowski T and Piedbois P: Efficacy of oral adjuvant therapy after resection of colorectal cancer: 5-year results from three randomized trials. J Clin Oncol 22: 484-492, 2004.

15. Sadahiro S, Suzuki T, Ishikawa K, Nakamura T, Tanaka Y, Masuda T, Mukoyama S, Yasuda S, Tajima T, Makuuchi H and Murayama C: Recurrence patterns after curative resection of colorectal cancer in patients for a minimum of ten years. Hepatogastroenterology 50: 1362-1366, 2003. 